Acta Crystallographica Section A

Foundations of Crystallography

ISSN 0108-7673

Received 4 August 2005

Accepted 9 November 2005

(C) 2006 International Union of Crystallography Printed in Great Britain - all rights reserved

\section{Quartz aggregates revisited}

\author{
Hans Grimmer \\ Laboratory for Development and Methods, Condensed Matter Research with Neutrons and Muons, \\ Paul Scherrer Institut, CH-5232 Villigen PSI, Switzerland. Correspondence e-mail: \\ hans.grimmer@psi.ch
}

Quartz aggregates that formed by coalescence of quartz crystals in the magma or in hydrothermal solution are considered. If the individuals have rhombohedral faces in contact, there will be two special cases: parallel intergrowths and intergrowths that agree in orientation and contact plane with Esterel twins grown from a twinned nucleus. For all other known cases, i.e. when the relative orientation satisfies the Japan, Sardinian, Tiflis, Zyndel-A or Samshvildo law, both individuals have exactly coinciding short symmetry translations in only one direction in the contact plane (monoperiodic twins). If a rhombohedral face is in contact with a prism face, monoperiodic twins will occur if the relative orientation satisfies the Zinnwald, Disentis or a proposed hypothetic law. The orientation of the two lattices can be expressed by a $180^{\circ}$ rotation about an axis with low indices independent of $c / a$ in the case of the Esterel, Japan and Sardinian laws. The same is true for the Tiflis and Zyndel-A laws only if they are redefined, and not at all in the case of the Samshvildo, Zinnwald, Disentis and hypothetic laws. When the two individuals have rhombohedral faces in contact, there will even be exact two-dimensional coincidence (multiplicity $\sigma$ ) in the contact plane and exact three-dimensional coincidence (multiplicity $\Sigma$ ) in space if the square of the axial ratio $c / a$ is rational. Indications are found that (despite their high values) these multiplicities may be related to the frequency of occurrence of intergrowths in low- and high-quartz.

\section{Introduction}

The stable modification of $\mathrm{SiO}_{2}$ at room temperature is $\alpha$-quartz (trigonal, space group $P 3_{2} 21$ or $P 3_{1} 21$ for right- or left-handed crystals, respectively). At elevated temperatures, it undergoes a reversible phase transition to $\beta$-quartz (hexagonal, space group $P 6_{2} 22$ or $P 6_{4} 22$ ). The transition temperature is $846 \mathrm{~K}$ at ambient pressure and is raised by $1 \mathrm{~K}$ per 40 bar of pressure increase (see Fig. 1). Almost all silica found in nature at ambient temperature and pressure is $\alpha$-quartz. Quartz originally formed as $\alpha$-quartz will be referred to as low-quartz, and quartz originally formed as $\beta$-quartz as high-quartz. Characteristic differences between low- and highquartz are found in the morphology and in the occurrence of twinning.

The prism $m\{10 \overline{1} 0\}$ and the positive and negative rhombohedra $r\{10 \overline{1} 1\}$ and $z\{01 \overline{1} 1\}$ dominate the habit of $\alpha$-quartz. They consist of six faces each. In $\beta$-quartz the 12 faces of $r$ and $z$ become equivalent and form the hexagonal dipyramid, which often is the only form occurring in $\beta$-quartz. In the following, we shall mainly consider aggregates that formed by coalescence of quartz crystals in the magma or in hydrothermal solution (Friedel, 1933; Laemmlein, 1940). In the case of high-quartz, it is then most probable that the contact plane between two individuals belongs to the hexagonal dipyramid in both of them.

In all aggregates in which $\{10 \overline{1} 1\}$ faces of both individuals are in contact, the lattices of the two individuals can be related by a rotation about the normal to (10ī1). Its angle $\varphi$ may be constrained to $0 \leq \varphi \leq 180^{\circ}$. The frequency of occurrence of

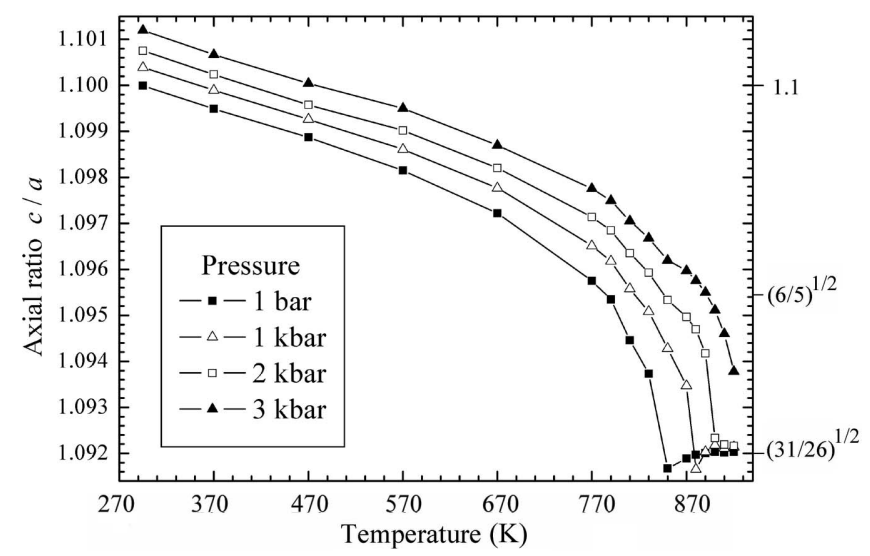

Figure 1

The axial ratio $c / a$ of quartz as a function of temperature and pressure. 
the $\varphi$ values is far from uniform and different for high- and low-quartz, as we shall discuss in the following.

\section{The net of the quartz lattice in (1011) coincidence nets}

Quartz has a hexagonal lattice. Its axial ratio $c / a$ is 1.100 for $\alpha$-quartz at ambient conditions and 1.092 for $\beta$-quartz, for which $c / a$ depends very little on temperature and pressure (see Fig. 1). The net in the (1011) plane is orthogonal centred $(o c)$ with axial ratio $b / a$ given by $b^{2}=3 a^{2}+4 c^{2}$. It follows that $b=2.8 a$ if $c=1.1 a$. An excellent approximation to the axial ratio of $\beta$-quartz is $c / a=(31 / 26)^{1 / 2} \simeq 1.0919$, for which $b / a=(101 / 13)^{1 / 2}$.

A rotation of a net (i.e. a two-dimensional lattice) about one of its lattice points by $\varphi=180^{\circ}$ is a symmetry operation of the net. Rotations with $0<\varphi<180^{\circ}$ about a lattice point can map a finite fraction $1 / \sigma$ of lattice points on lattice points of the $o c$ net only if $(b / a)^{2}$ is rational (Fortes, 1977). If this is the case, then the points common to the original and the rotated net also form a two-periodic net, called the coincidence net; $\sigma$ is a positive integer, called the multiplicity of the coincidence net. The multiplicities $\sigma$ are equal for $\varphi$ and for $180^{\circ}-\varphi$.

\section{High-quartz aggregates with contact plane $\{10 \overline{1} 1\}_{1} \|\{10 \overline{1} 1\}_{2}$}

Laemmlein (1940) describes aggregates of quartz crystals in quartz porphyrs from the Caucasus (Samshvildo in Georgia and Kafan in Armenia), in which the contact plane is of type $\{10 \overline{1} 1\}$ for both individuals involved. This paper being in Russian (with a summary in English) and published in a journal that is not easily accessible, I shall summarize results that are of importance in the sequel. Laemmlein observed that weathered $\{10 \overline{1} 1\}$ surfaces with typical size $25 \mathrm{~mm}^{2}$ of quartz crystals from quartz porphyrs often contained shining fresh parts with typical size $1 \mathrm{~mm}^{2}$. The large weathered surfaces and these small shining parts both had the form of isosceles triangles with the same length ratio, showing that the shining parts correspond to $\{10 \overline{1} 1\}$ planes of smaller crystals that had been in contact with the large ones (Figs. 2 and 1 in Laemmlein, 1940). Measuring the orientation of the small triangle relative to the large one, he determined the absolute value $\varphi$ of the angle about the normal to the contact plane by which the lattice of the small crystal was rotated with respect to the lattice of the large one and obtained the results given in the first two columns of Table 1. Column 3 gives the names of the twin laws for which the lattices of the two twin components are related by the rotation in column 2. According to Frondel (1962), Esterel twins in high-quartz consist of two crystals of approximately equal size and with contact plane usually parallel to $\{10 \overline{1} 1\}$. The large difference in size of adjacent crystals observed by Laemmlein makes it very unlikely that they developed from a twinned nucleus. This is even more evident if we consider Verespatak twins, which according to Frondel (1962) have contact plane $\{11 \overline{2} 2\}$, not $\{10 \overline{1} 1\}$ as in the aggregates observed by Laemmlein (1940). Laemmlein,
Table 1

Results of Laemmlein (1940) concerning high-quartz aggregates with contact plane $\{10 \overline{1} 1\}_{1} \|\{10 \overline{1} 1\}_{2}$.

\begin{tabular}{|c|c|c|}
\hline $\begin{array}{l}\text { Number } \\
\text { of imprints } \\
\text { (total 672) }\end{array}$ & $\begin{array}{l}\text { Rotation angle } \varphi\left({ }^{\circ}\right) \\
\text { about the normal } \\
\text { to }\{10 \overline{1} 1\}\end{array}$ & $\begin{array}{l}\text { Name of corresponding } \\
\text { twin law }\end{array}$ \\
\hline 192 & 0 & [Parallel intergrowth] \\
\hline 216 & 180 & Esterel \\
\hline 131 & 140.7 & Verespatak (=Japan) \\
\hline 37 & 39.3 & 'Samshvildo' \\
\hline 10 & Others & Sardinian, Zyndel-A, Tiflis, ... \\
\hline 86 & $\begin{array}{c}\text { Impossible to } \\
\text { determine }\end{array}$ & \\
\hline
\end{tabular}

therefore, explains the formation of his aggregates by the fusion of single crystals in their mother liquid. He also discusses features of the shining fresh parts that give indications of the attachment mechanism, a short version of which is given in the English summary of his paper.

Laemmlein (1940) proposed the name 'Samshvildo twins' for his aggregates with $\varphi=39.3^{\circ}$; I shall show below that this relative orientation of lattices does not correspond to a genuine twin law. Notice that the overwhelming majority of imprints (576 of the 586 whose orientation could be determined) have $\varphi=0$ or $140.7^{\circ}$ or their supplements $(180-0)^{\circ}$, $(180-140.7)^{\circ}$. Laemmlein (1940) also mentions the Sardinian, Zyndel-A and Tiflis laws but does not give details of their occurrence because the corresponding imprints were not clear. The rare occurrence of $\varphi$ values other than $0,180,140.7$ and $39.3^{\circ}$ and the vagueness of the corresponding imprints show that the forces holding the two $\{10 \overline{1} 1\}$ surfaces together are weak in these cases. An example of a high-quartz aggregate with contact plane as given above and relative orientation of the two individuals corresponding to the Sardinian law has been described by Drugman (1922).

The lowest possible values of the multiplicity $\sigma$ for the $o c$ net with $b / a=(101 / 13)^{1 / 2}$ are given in Table 2 .

For high-quartz, it follows from Tables 1 and 2 that (10 $\overline{1} 1)$ intergrowths with $\sigma=1$ occur most frequently, those with $\sigma=57$ are still quite frequent, whereas those with higher values of $\sigma$ are very rare. The last column in Table 2 gives the threedimensional coincidence index $\Sigma$. This column shows that for one of the angles $\varphi$ there is coincidence in every lattice plane (1011), whereas for its supplement, $180^{\circ}-\varphi$, coincidence occurs only in one out of 101 lattice planes (10i1). The aggregates in Table 2 with $\Sigma=\sigma$ and $\sigma>1$ may be considered as plesiotwins according to their definition by Nespolo et al. (1999); an alternative interpretation as monoperiodic twins will be discussed below.

It should be noticed that Esterel and Verespatak (Japan) twins occur in high-quartz often as growth twins, i.e. twins grown from a twinned nucleus (see Frondel, 1962). The usual contact plane is then (1011) for Esterel twins but (112̄2) for Verespatak (Japan) twins. Remember that only the first contact plane is considered by Laemmlein (1940).

The relative orientation of the lattices of the two individuals can be described by a mirror reflection in $\{101\}$ or by a $180^{\circ}$ 
Table 2

The lowest possible multiplicity values $\sigma$ of the coincidence net in the contact plane for $b / a=(101 / 13)^{1 / 2}$.

\begin{tabular}{|c|c|c|c|c|}
\hline$\sigma$ & $\varphi\left({ }^{\circ}\right)$ & Name of the orientation & $\begin{array}{l}\text { Indices of the } \\
\text { corresponding } 180^{\circ} \\
\text { rotation axis }\end{array}$ & $\Sigma$ \\
\hline \multirow[t]{2}{*}{1} & 0 & [Parallel intergrowth] & & $\sigma$ \\
\hline & 180 & Esterel & [212] & $101 \sigma$ \\
\hline \multirow[t]{2}{*}{57} & 39.47 & Samshvildo & {$[57,44,13]$} & $101 \sigma$ \\
\hline & 140.53 & Verespatak (=Japan) & [111] & $\sigma$ \\
\hline \multirow[t]{2}{*}{109} & 85.79 & Sardinian & [211] & $\sigma$ \\
\hline & 94.21 & - & {$[70,39,39]$} & $101 \sigma$ \\
\hline \multirow[t]{2}{*}{153} & 71.32 & - & {$[127,75,52]$} & $101 \sigma$ \\
\hline & 108.68 & Tiflis & [322] & $\sigma$ \\
\hline \multirow[t]{2}{*}{213} & 58.28 & - & [321] & $\sigma$ \\
\hline & 121.72 & - & {$[83,65,65]$} & $101 \sigma$ \\
\hline \multirow[t]{2}{*}{309} & 69.74 & Zyndel-A & [532] & $\sigma$ \\
\hline & 110.26 & - & {$[153,104,104]$} & $101 \sigma$ \\
\hline
\end{tabular}

rotation about $\langle 212\rangle$ in an Esterel (Reichenstein-Grieserntal) twin, by a mirror reflection in $\{102\}$ or by a $180^{\circ}$ rotation about $\langle 211\rangle$ in a Sardinian twin, and by a mirror reflection in $\{112\}$ or by a $180^{\circ}$ rotation about $\langle 111\rangle$ in a Verespatak (Japan) twin for $\beta$ - (and $\alpha$-) quartz, i.e. independent of the axial ratio cla (see e.g. Frondel, 1962). Laemmlein (1940) defined the Samshvildo orientation by having its rotation angle $\varphi$ equal to $180^{\circ}$ minus the rotation angle for the Verespatak (Japan) orientation. The relative orientation of the lattices of the two individuals can then be described by a mirror reflection in $(70,31,31)$ or by a $180^{\circ}$ rotation about $[57,44,13]$ if $c / a=(31 / 26)^{1 / 2}(\beta$-quartz $)$ and by a mirror reflection in $(271,121,121)$ or by a $180^{\circ}$ rotation about $[221,171,50]$ if $c / a=1.1$ ( $\alpha$-quartz). This shows that neither a mirror reflection in a plane with low indices nor a $180^{\circ}$ rotation about an axis with low indices can describe the Samshvildo orientation, which, therefore, does not correspond to a genuine twin orientation. The situation is similar if the angle $\varphi$ corresponding to the Sardinian, Tiflis or Zyndel-A orientation is replaced by its supplement, $180^{\circ}-\varphi$.

\section{Low-quartz aggregates with contact plane $\{10 \overline{1} 1\}_{1} \|\{10 \overline{1} 1\}_{2}$}

Aggregates with contact plane $\{10 \overline{1} 1\}_{1} \|\{10 \overline{1} 1\}_{2}$ occur not only in high-quartz but also in low-quartz, as shown in the review by Frondel (1962). Is the frequency of the relative orientation of the two components of such aggregates different for lowand high-quartz?

In his paper 'Über Quarzzwillinge mit nichtparallelen Hauptaxen' (On quartz twins with non-parallel principal axes), Zyndel (1913) describes two laws with contact plane (1011) for both individuals: Reichenstein-Grieserntal (= Esterel) and Sardinian and postulates two others, examples of which have been found in the meantime in low-quartz and are now known as the Tiflis and Zyndel-A law, respectively (see Frondel, 1962). For the Sardinian law, Zyndel refers to the only example known to him, described by Sella (1858), who
Table 3

The lowest possible multiplicity values $\sigma$ of the coincidence net in the contact plane for $b / a=2.8$.

\begin{tabular}{|c|c|c|c|c|}
\hline$\sigma$ & $\varphi\left({ }^{\circ}\right)$ & Name of the orientation & $\begin{array}{l}\text { Indices of the } \\
\text { corresponding } 180^{\circ} \\
\text { rotation axis }\end{array}$ & $\Sigma$ \\
\hline \multirow[t]{2}{*}{1} & 0 & [Parallel intergrowth] & & $\sigma$ \\
\hline & 180 & $\begin{array}{l}\text { Reichenstein-Grieserntal } \\
\quad(=\text { Esterel })\end{array}$ & [212] & $196 \sigma$ \\
\hline 140 & 90 & & {$[19,10,10]$} & $14 \sigma$ \\
\hline \multirow[t]{2}{*}{148} & 71.08 & - & {$[123,73,50]$} & $98 \sigma$ \\
\hline & 108.92 & Tiflis & [322] & $2 \sigma$ \\
\hline \multirow[t]{2}{*}{149} & 69.98 & Zyndel-A & [532] & $4 \sigma$ \\
\hline & 110.02 & - & {$[37,25,25]$} & $49 \sigma$ \\
\hline \multirow[t]{2}{*}{175} & 53.13 & & & \\
\hline & 126.87 & & & \\
\hline \multirow[t]{2}{*}{203} & 43.60 & & & \\
\hline & 136.40 & & & \\
\hline \multirow[t]{2}{*}{221} & 39.31 & Samshvildo & {$[221,171,50]$} & $196 \sigma$ \\
\hline & 140.69 & Japan & [111] & $\sigma$ \\
\hline \multicolumn{5}{|l|}{265} \\
\hline \multicolumn{5}{|l|}{287} \\
\hline \multicolumn{5}{|l|}{325} \\
\hline \multicolumn{5}{|l|}{364} \\
\hline \multirow[t]{2}{*}{421} & 86.05 & Sardinian & {$[211]$} & $\sigma$ \\
\hline & 93.95 & - & {$[271,150,150]$} & $196 \sigma$ \\
\hline
\end{tabular}

examined an example of unknown origin, of which it is not clear whether it was low- or high-quartz (cf. Drugman, 1922 and Frondel, 1962). I am not aware of an aggregate with contact plane (1011) in both individuals, whose orientation corresponds to the Sardinian law and which is clearly lowquartz.

The lowest possible values of the multiplicity $\sigma$ for the $o c$ net with $b / a=2.8$ are given in Table 3. The $\sigma$ values in Table 3 are roughly the same as those in Table 2 for the Tiflis orientation, two times smaller for the Zyndel-A orientation, but four times larger for the Samshvildo, Japan and Sardinian orientations. This may suggest that aggregates with contact plane (1011) occur in low-quartz more frequently in Zyndel-A or Tiflis orientation than in Japan, Samshvildo or Sardinian orientation. The experimental situation is less clear than in the case of high-quartz: I am not aware of a recent survey of aggregates with contact plane (1011) in low-quartz; the axial ratio depends much more strongly on temperature and pressure for $\alpha$-quartz than for $\beta$-quartz. Fig. 1 gives the dependence of $c / a$ on temperature and pressure according to the results of Girsperger et al. (2002).

According to the definitions given by Zyndel (1913) and reproduced by Frondel (1962), the planes (10i1) of both individuals are parallel in Tiflis twins and in Zyndel-A twins; in addition, $[010]_{1} \|[10 \overline{1}]_{2}$ and $[\overline{1} \overline{1} 1]_{1} \|[010]_{2}$ for Tiflis; $[\overline{101}]_{1} \|[010]_{2}$ and $[010]_{1} \|[11 \overline{1}]_{2}$ for Zyndel-A. This is possible only for $c / a=(5 / 4)^{1 / 2}$, in which case $\varphi=109.47^{\circ}$ for Tiflis and $180-109.47^{\circ}=70.53^{\circ}$ for Zyndel-A. The situation for $c / a=$ $(5 / 4)^{1 / 2}=1.118$, which clearly lies outside the experimental range for quartz, is shown in Fig. 2.

For $c / a=(5 / 4)^{1 / 2}$, the relative orientation of the lattices of two individuals can be described by a $180^{\circ}$ rotation about [416] for a Tiflis twin and by a $180^{\circ}$ rotation about [716] for a 
Zyndel-A twin. These indices, being reasonably low, will be retained for axial ratios in the experimental range of quartz. It follows then that coincidence is retained along the directions $\mathbf{u}+\mathbf{v}$ in Fig. 2, whereas the coincidences along $\mathbf{u}$ and along $\mathbf{v}$ are no longer exact, and the sum of the $\varphi$ values for Tiflis and for Zyndel-A differs from $180^{\circ}$ if $c / a \neq(5 / 4)^{1 / 2}$. Exact coincidence in the contact plane is then one-dimensional, except for rational values of $(c / a)^{2}$, for which the two-dimensional coincidence density $1 / \sigma$ varies widely with $(c / a)^{2}$, as shown in Table 4.

Also, if the two individuals meeting in a common (10 $\overline{1} 1)$ plane have a relative orientation given by the Japan or Sardinian law, it follows from their usual definition that there is one-dimensional coincidence for any value of $c / a$ and twodimensional coincidence only for rational values of $(c / a)^{2}$. The situation is illustrated in Fig. 3 for $c / a=(6 / 5)^{1 / 2}$.

\section{Aggregates with contact plane $\{10 \overline{1} 0\}_{1} \|\{10 \overline{1} 1\}_{2}$}

The best known example of this type is the Zinnwald twin, for which $[010]_{1} \|[010]_{2}$ (Zyndel, 1913; Drugman, 1927, 1930; Friedel, 1933). In this case, one obviously has one-dimensional coincidence along [010] for any value of $c / a$. The area of primitive cells is $A_{1}=a c$ in $\{10 \overline{1} 0\}$ and $A_{2}=a\left(\frac{3}{4} a^{2}+c^{2}\right)^{1 / 2}$ in $\{10 \overline{1} 1\}$.

If $c / a=1.1$, then $A_{1}=1.1 a^{2}, A_{2}=1.4 a^{2}$, and one even has two-dimensional coincidence in the contact plane with $\sigma_{1}=28$ and $\sigma_{2}=22$ and three-dimensional coincidence in space with $\Sigma=308$ (see Grimmer \& Kunze, 2004). The coincidence cell in the contact plane is of type op with side lengths $a$ and 28c; the three-dimensional coincidence cell is of type $o F$.

Zyndel (1913) discusses several intergrowths related to Zinnwald by an additional rotation of individual two by an angle $\alpha$ about the normal to the contact plane. Before discussing two of them from the coincidence point of view, we point out that it follows for $c / a=1.1$ from the values of $A_{1}$ and

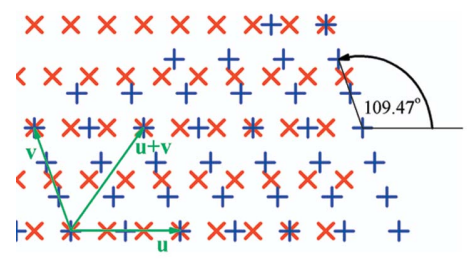

\section{Tiflis}

u: $\left[\begin{array}{lll}0 & 3 & 0\end{array}\right]_{1}=\left[\begin{array}{lll}2 & 0 & 2\end{array}\right]_{2}$

$\mathbf{v}:\left[\begin{array}{lll}\overline{2} & \overline{2} & 2\end{array}\right]_{1}=\left[\begin{array}{lll}0 & 3 & 0\end{array}\right]_{2}$

It follows that

$\mathbf{u}+\mathbf{v}:\left[\begin{array}{lll}\overline{2} & 1 & 2\end{array}\right]_{1}=\left[\begin{array}{lll}2 & 3 & 2\end{array}\right]_{2}$

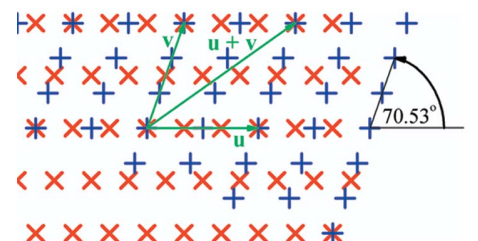

Zyndel-A

u: $\left[\begin{array}{lll}0 & 3 & 0\end{array}\right]_{1}=\left[\begin{array}{lll}2 & 2 & 2\end{array}\right]_{2}$

v: $\left[\begin{array}{lll}\overline{2} & 0 & 2\end{array}\right]_{1}=\left[\begin{array}{lll}0 & 3 & 0\end{array}\right]_{2}$

It follows that

$\mathbf{u}+\mathbf{v}:\left[\begin{array}{lll}\overline{2} & 3 & 2\end{array}\right]_{1}=\left[\begin{array}{lll}2 & 5 & 2\end{array}\right]_{2}$

$\times \times \times \times \times \times \times \times$ 米

Figure 2

The nets of lattice points in the contact plane for Tiflis and Zyndel-A orientations and an axial ratio $c / a=(5 / 4)^{1 / 2}$. Every sixth point of the red nets and every sixth point of the blue nets coincide, i.e. $\sigma=6$.
Table 4

The multiplicities $\sigma$ for a number of orientations and values of the axial ratio $c / a$.

\begin{tabular}{lrrrr}
\hline & $(c / a)^{2}$ & & \\
\cline { 2 - 5 } $\begin{array}{l}\text { Relative } \\
\text { orientation }\end{array}$ & $31 / 26=1.1923$ & $6 / 5=1.2$ & $121 / 100=1.21$ & $5 / 4=1.25$ \\
\hline Esterel & 1 & 1 & 1 & 1 \\
Tiflis & 153 & 59 & 148 & 6 \\
Zyndel-A & 309 & 119 & 149 & 6 \\
Japan/Samshvildo & 57 & 11 & 221 & 9 \\
Sardinian & 109 & 7 & 421 & 17 \\
\hline
\end{tabular}

$A_{2}$ that $\sigma_{1}=14 m, \sigma_{2}=11 m, \Sigma=154 m n$, where $m$ and $n$ are integers. [In our examples, $n=1$ and $m=2$ (Zinnwald), 5 (Seedorf II), 13 (Disentis) or 17 (hypothetic).]

Seedorf II is defined by $[001]_{1} \|[010]_{2}$, i.e. it is obtained by a rotation with $\alpha=90^{\circ}$ for any value of $c / a$. In general, there is not even one-dimensional coincidence in the contact plane; however, if $c / a=1.1$, we have two-dimensional coincidence in the contact plane with $\sigma_{1}=70$ and $\sigma_{2}=55$ and three-dimensional coincidence in space with $\Sigma=770$; the coincidence cell in the contact plane is of type $o c$ with side lengths $10 a$ and $14 c$. If $c / a=1.1$, the two lattices are not related by a $180^{\circ}$ rotation and, therefore, not by a mirror reflection.

Disentis is defined by $[01 \overline{1}]_{1} \|[11 \overline{1}]_{2}$. These two vectors lie in the contact plane and have the same length for any value of $c / a$, so that we have at least one-dimensional coincidence in the contact plane. If $c / a=1.1$, then $\sin \alpha=5 / 13, \alpha=22.62^{\circ}$ and one even has two-dimensional coincidence in the contact plane with $\sigma_{1}=182$ and $\sigma_{2}=143$ and three-dimensional coincidence in space with $\Sigma=2002$.

There is a similar hypothetic possibility, not discussed by Zyndel, which is defined by $[01 \overline{1}]_{1} \|[10 \overline{1}]_{2}$. Also these two vectors lie in the contact plane and have the same length for any value of $c / a$, so that we have at least one-dimensional coincidence. If $c / a=1.1$, then $\sin \alpha=15 / 17, \alpha=61.93^{\circ}$ and one even has two-dimensional coincidence in the contact plane with $\sigma_{1}=238$ and $\sigma_{2}=187$ and three-dimensional coincidence in space with $\Sigma=2618$.
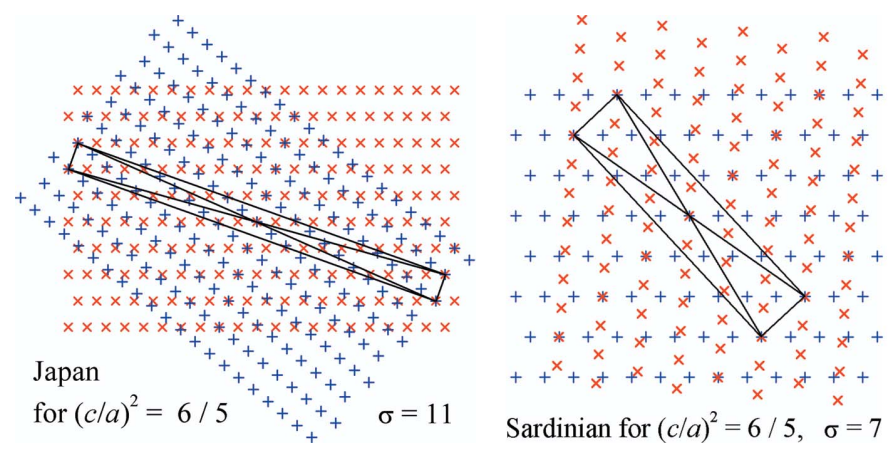

Figure 3

The nets of lattice points in the contact plane (1011) for intergrowths in Japan and Sardinian orientations and an axial ratio $c / a=(6 / 5)^{1 / 2}$. 
The contact planes in Disentis orientation and in our hypothetic one are shown in Fig. 4 for $c / a=1.1$.

Zyndel (1913) mentions the Zinnwald, Seedorf II and Disentis orientations in connection with low-quartz. Exact three-dimensional coincidence occurs for $c / a=1.1$, but not for $(c / a)^{2}=31 / 26$ or 6/5. As stated above, the Zinnwald, Disentis and our hypothetic laws lead to one-dimensional coincidence for any value of $c / a$. We conclude that these laws may occur also in high-quartz; the dense coincidence along a row being of prime importance. In fact, the Zinnwald orientation has been found also in high-quartz (Drugman, 1927, 1930).

\section{Discussion}

Considering aggregates of two quartz crystals having as contact plane either $\{10 \overline{1} 1\}_{1}$ or $\{10 \overline{1} 0\}_{1}$ parallel to $(10 \overline{1} 1)_{2}$, we discussed a number of possible orientation relations of the crystal lattices of the two individuals, corresponding to the Japan, Samshvildo, Sardinian, Tiflis and Zyndel-A laws in the first case, and to the Zinnwald, Disentis and hypothetic laws in the second. In all these cases and for any value of $c / a$ in the experimental range of quartz, there is a row in the contact plane that, in both individuals, has small indices and the same small period. This period is given in Table 5 .

Specification of a row in individual 1 that is parallel to a row in individual 2 does not uniquely determine the relative orientation of the lattices of the two individuals. The orientation becomes unique only when also the indices of the contact plane are specified in both individuals. Twins in which the lattices of the two components have coincident translation vectors in only one direction have been called monoperiodic (see Friedel, 1933; Hahn \& Klapper, 2003; Nespolo \& Ferraris, 2004).

Taking into account that, in most cases, two individuals of different size are in contact, the small one occupying only part of the contact plane of the large one, it is plausible that such aggregates formed when the two individuals had already reached macroscopic size and met in the magma or hydrothermal solution. Such a mechanism has been proposed by Drugman (1930) and by Friedel (1933) for the Zinnwald case and by Laemmlein $(1930,1940)$ for the cases considered in
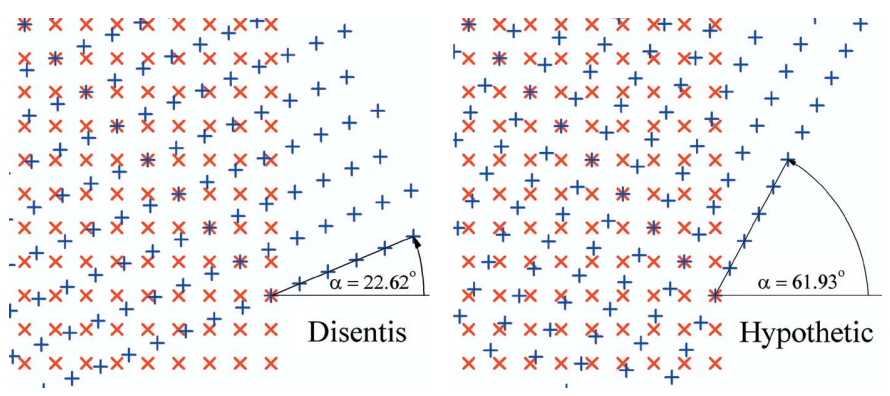

Figure 4

The nets of lattice points in the contact plane (1011) for an axial ratio $c / a=1.1$ for intergrowths in Disentis and our hypothetic orientation.
Table 5

The period of one-dimensional coincidence in the contact plane.

\begin{tabular}{llll}
\hline$(10 \overline{1} 1)_{2} \|$ & Orientation & Square of period & Period if $c^{2} / a^{2}=6 / 5$ \\
\hline$\{10 \overline{1} 1\}_{1}$ & Japan & $a^{2}+c^{2}$ & $1.48 a$ \\
& Sardinian & $3 a^{2}+c^{2}$ & $2.05 a$ \\
& Tiflis & $7 a^{2}+4 c^{2}$ & $3.44 a$ \\
$\multirow{2}{*}{10 \overline{1} 0}_{1}$ & Zyndel-A & $19 a^{2}+4 c^{2}$ & $4.88 a$ \\
& Zinnwald & $a^{2}$ & $a$ \\
& Disentis & $a^{2}+c^{2}$ & $1.48 a$ \\
& Hypothetic & $a^{2}+c^{2}$ & $1.48 a$ \\
\hline
\end{tabular}

\$3. A recent review of the oriented attachment mechanism is due to Nespolo \& Ferraris (2004). This review summarizes also the experiment of Schaskolsky \& Schubnikow (1933), who artificially produced intergrowths of potassium alum $\left[\mathrm{KAl}\left(\mathrm{SO}_{4}\right)_{2}\left(\mathrm{H}_{2} \mathrm{O}\right)_{12}\right]$ in aqueous solution in order to test the oriented attachment mechanism suggested by Laemmlein (1930). They grew octahedral alum crystals of size $20-30 \mathrm{~mm}$ and let octahedral- and cube-shaped crystals of size $\leq 0.5 \mathrm{~mm}$ fall onto them. Strong shaking separated most small crystals from the large ones. Measuring the orientation of those that remained stuck, they found that some orientations occurred much more frequently than at random. For small octahedral crystals on a large octahedral one, the orientations with $\sigma=1$, i.e. parallel orientation $(\Sigma=1)$ and spinel orientation $(\Sigma=3)$, occurred preferentially, similarly to parallel and Esterel orientations in high-quartz ( $\$ 3)$. For small cube-shaped crystals on the large octahedral one, the orientations with parallel $\langle 110\rangle$ directions in the two planes in contact occurred preferentially; in this case, which resembles Zinnwald twins in quartz (§5), the coincidence is one-dimensional.

For rational values of $(c / a)^{2}$, the coincidence in the contact plane $\{10 \overline{1} 1\}_{1} \|\{10 \overline{1} 1\}_{2}$ becomes two-dimensional, although with a very large cell except in the cases illustrated in Fig. 3. We showed that, nevertheless, there are indications that the frequency of occurrence of aggregates with contact plane $\{10 \overline{1} 1\}_{1} \|\{10 \overline{1} 1\}_{2}$ in high- and low-quartz, respectively, is correlated with the values of $\sigma$ and $\Sigma$, which give the inverse density of coincidence sites in the contact plane and in space, respectively.

The situation is different for the Esterel orientation: also Esterel twins grown from a twinned nucleus have contact planes of type $\{10 \overline{1} 1\}$ in both individuals. All the nodes of the net in the contact plane are in coincidence for any value of $c / a$. Aggregates in Esterel orientation with contact plane $\{10 \overline{1} 1\}_{1} \|\{10 \overline{1} 1\}_{2}$ may have originated from twinned nuclei or by aggregation of preformed crystals, as was pointed out already by Friedel (1933).

There remains the Seedorf II law, observed in low-quartz (Zyndel, 1913, Frondel, 1962). In this case, there is no dense coincidence along a row in the contact plane. For $c / a=1.1$, the values of $\sigma_{1}$ and $\sigma_{2}$ are 2.5 times larger than the lowest possible ones for the contact plane $\{10 \overline{1} 0\}_{1} \|\{10 \overline{1} 1\}_{2}$. To our knowledge, the Seedorf II orientation has been described only once, so that its occurrence with more than random probability has not been established. 


\section{Conclusions}

In intergrowths of high-quartz with contact plane (1011), the frequency of occurrence of the angle $\varphi$ of the rotation about the normal to $(10 \overline{1} 1)$ has pronounced maxima at those values that correspond to the largest possible densities of coincidence sites in the contact plane. The angles $\varphi$ and $180^{\circ}-\varphi$ give rise to the same density $1 / \sigma$ in the contact plane but to different three-dimensional coincidence densities $1 / \Sigma$. The orientation with the lower value of $\Sigma$ appears more frequently and corresponds to an orientation observed in twins growing from a twinned nucleus, the other does not correspond to such a twin, e.g. Japan versus Samshvildo. Similarly, the Tiflis and Zyndel-A orientations are not supplementary, in contrast to the idea of Zyndel (1913).

Based on the results of Laemmlein (1940) (and Zyndel, 1913), we showed that monoperiodic twins in quartz are rather common in aggregates formed by coalescence of preformed quartz crystals in the magma or in hydrothermal solution and are by no means restricted to Zinnwald-type orientation.

Stimulating discussions with K. Kunze and M. Nespolo are gratefully acknowledged.

\section{References}

Drugman, J. (1922). Mineral. Mag. 19, 295-300.

Drugman, J. (1927). Mineral. Mag. 21, 366-382.

Drugman, J. (1930). Bull. Soc. Fr. Minéral. 53, 95-104.

Fortes, M. A. (1977). Phys. Status Solidi B, 82, 377-382.

Friedel, G. (1933). Bull. Soc. Fr. Minéral. 56, 262-274.

Frondel, C. (1962). The System of Mineralogy, Vol. III. New York: Wiley.

Girsperger, S., Raz, U. \& Thompson, A. B. (2002). ETH E-Collection, http://e-collection.ethbib.ethz.ch/show?type=bericht\&nr=184.

Grimmer, H. \& Kunze, K. (2004). Acta Cryst. A60, 220-232.

Hahn, Th. \& Klapper, H. (2003). International Tables for Crystallography, Vol. D, Physical Properties of Crystals, edited by A. Authier, ch. 3.3, pp. 393-448. Dordrecht: Kluwer Academic Publishers.

Laemmlein, G. G. (1930). Dokl. Akad. Nauk SSSR, pp. 709-714. (In Russian.)

Laemmlein, G. G. (1940). Trav. Lab. Cristallogr. Acad. Sci. URSS, No. 2, pp. 123-148. (In Russian.)

Nespolo, M. \& Ferraris, G. (2004). Eur. J. Mineral. 16, 401-406.

Nespolo, M., Ferraris, G., Takeda, H. \& Takéuchi, Y. (1999). Z. Kristallogr. 214, 378-382.

Schaskolsky, M. \& Schubnikow, A. (1933). Z. Kristallogr. 85, $1-16$.

Sella, Q. (1858). Mem. R. Accad. Sci. Torino, Ser. 2, 17, 321-332, pl. V, Figs. 48-50.

Zyndel, F. (1913). Z. Kristallogr. 53, 15-52. 